\title{
A study of sector configurations capacity for air traffic service
}

\author{
Teodosiy Todorov ${ }^{1, *}$, and Plamen Petrov \\ ${ }^{1}$ Technical University - Sofia, Department of Air Transport, 8 Kliment Ohridski Blvd., 1000 Sofia, Bulgaria
}

\begin{abstract}
A study of sector configurations capacity in the upper air space of the Republic of Bulgaria is presented. The method used to determine the capacity of sectoral configurations is based on the air traffic control workload. The results obtained can be used to assess and improve airspace capacity and for efficient planning and use of air traffic service resources.
\end{abstract}

\section{Introduction}

In the literary sources there are definitions for Airspace Capacity, which differ in their essence. Capacity is the maximum number of aircraft which can be serviced for a particular period of time by the system or one of its components [1]. The capacity of the Air Traffic Management (ATM) system is the maximum number of aircraft which can be simultaneously serviced safely and efficiently, while the Air Traffic Controller (ATC) is experiencing acceptable workload [2]. According to others, capacity is defined by the maximum number of aircraft which can be serviced in a given sector for ATS for a particular period of time while the ATC is experiencing his/her upper limit of workload [3]. Each ATM system evaluates the degree of workload its airspace can handle. This is the reason why there are so many models developed for determining the capacity which must meet the needs of every Air Navigation Service Provider (ANSP). We can summarize that all the methods are based on the workload of ATCs.

The capacity of airspace acquires new aspects and timeliness in free route airspace (FRA). As a result, the character of air traffic flows will change in airspace in each country. For this, it is necessary to assess air traffic, which can be serviced by each ATS sector.

\section{Task}

The present task is to study the capacity of sector configurations in the Bulgarian Flight Information Region (FIR). Examination of the capacity of sector configurations in the Upper Airspace.

The range of the study is limited to reviewing the workload of an ATC for allocating the capacity of sector configurations of family sectors Varna. The task is to select the categories of work tasks decided by the ATCOs and to define the capacity of an ATS sector. Statistical data from the Eurocontrol database have been used for the purpose of the study.

The study is conducted for airspace, consisting of four sectors - VA, VB, VC, and VD [4], with limits to their upper boundaries up to flight level (FL) 450. The airspace is configured in different sector configurations, vertically and horizontally. In height, the airspace can be configured without division from FL 245 to FL 660, with one division at FL 245, or with two divisions at FL 345 and FL 375. Sector VC is not used by itself, but in a combination with different sectors. An average speed of $\mathrm{Vm}=460 \mathrm{kt}$ is defined for commonly used types of airspace for the time of the study.

\section{Literary Analysis}

A study of the literature shows that there are no documents pertaining to the dynamic management of the airspace. Some of the software provides functions related to the dynamic sectors division and should be applied. When calculating the ATM sector capacity different models and methods are used.

The DORATAK is an analytic method used for estimating working tasks and analysis of workload [5]. It is based on simulation (Fast-Time Simulation - FTS). An ATC's workload is defined by the time needed for executing "observed" and "non-observed" tasks, while the capacity is defined as the workload level by which the ATC has enough time for "recovery".

Another method is presented in [6]. The ATM sector capacity evaluation is the maximum number of aircraft which a single ATC can handle simultaneously in one sector. With this method different working tasks done by the ATC are considered all together.

The ATC Capacity Analyzer (CAPAN) method, is a Fast Time Simulation (FTS), developed by EUROCONTROL. This method uses an airspace model in which workload is generated for the simulated working position with given air traffic. After the exercise CAPAN evaluates the workload. The result is compared to a preset upper limit to traffic-handling capacity. In order to make the simulated model of airspace as similar to real airspace as possible, the correlation between qualitative (high and low workload) and quantitative evaluation is determined experimentally.

\footnotetext{
Corresponding author: teodosiy.todorov@bulatsa.com
} 
NEST is a software product for modeling through a set of scenarios, used by a EUROCONTROL Network Manager and an ANSP for design and development of airspace structure, capacity planning and analysis. NEST uses database for the European airspace and route network as well as traffic forecasts by database STATFOR, which are provided by EUROCONTROL.

The evaluation of the methods examined shows that they are not suitable for FRA.

\section{Macroscopic model of workload for capacity evaluation}

For the purposes of this study, a macroscopic model of workload for capacity assessment has been selected. The macroscopic model performs quantitative evaluation of workload depending on the geometry of the ATS sector, on the direction of air traffic flow and on the frequency of conflicts between airborne aircraft. This analytical approach is easily extrapolated in new conditions and its parameters can be selected and defined by comparison to real traffic.

Using this model, we can investigate the correlation between the capacity of the ATM sectors and the effectiveness of the air traffic controllers' work.

When a "J" number of separate tasks are present, the "intensity of the workload" takes the following form:

$$
G=\sum_{j=1}^{J} \tau_{j} \lambda_{j}
$$

where $\tau_{j} \mathrm{e}$ is the time necessary to fulfill a task $j$, while $\lambda_{j}$ is the rate at with which it is fulfilled.

The determined value Gm, at which an air traffic controller will no longer be able to perform his work safely if he has to handle additional traffic, is the maximum level of workload and determines the capacity of the ATM sector.

Using this model, the work tasks are summarized in four categories - background tasks, transition tasks, recurring tasks and conflict tasks.

The background tasks include routine actions and occupy a constant, small part of the air traffic controller's time. The workload from the background tasks can be presented in the following form:

$$
G_{b}=\tau_{b} \lambda_{b}
$$

Transition tasks are performed every time an aircraft passes through the sector. These tasks require a moderate amount of time to be performed $\tau_{t}$ and a moderate rate at which they are performed $\lambda_{t}$ that can be presented in the following way:

$$
\lambda_{t}=E[N] / T,
$$

where $E[N]$ is the average number of aircraft in a particular sector and $T$ is the average time it takes an aircraft to fly through the aforementioned sector.

Recurring tasks are performed periodically, while the observed aircraft is present within the particular ATM sector.
These tasks require an average amount of time to do $\tau_{r}$ and are performed at a rate of $\lambda_{r}$, where:

$$
\lambda_{r}=E[N] / P
$$

In this formula $P$ is the average time period, during which the tasks are performed.

For conflict tasks it is accepted, that an average amount of time $\tau_{c}$ is necessary for their completion and an average rate of completion $\lambda_{c}$. For a current sector with a defined number of aircraft $-N_{s}$, the rate of completion of conflict tasks can be presented as:

$$
\lambda_{c}=B N_{s}^{2} / Q
$$

where $B$ is the physical constant, dependent on the velocity of the converging speed between 2 aircraft and the separation norms; $N_{s}$ - number of aircraft; $Q$ - sector volume.

The number of aircraft $N$ in the considered sector is a random quantity and $\lambda_{c}$ is proportional to the expected value of $E[N]$. Representing the bulk density of aircraft as $k$ :

$$
k Q=E[N],
$$

and assuming the distribution of Poisson [2], the following frequency for conflict tasks is received:

$$
\lambda_{c}=B k(k Q+1)
$$

Substituting it in (1), it can be said that the intensity of the overall workload $G$ is the following:

$$
G=G_{b}+\tau_{t} k Q / T+\tau_{r} k Q / P+\tau_{c} B k(k Q+1) .
$$

For this method there is no need for a large computational resource or access to statistical information for the air traffic flow. An expert opinion is needed for the correct selection and determination of the empiric parameters.

\section{Determining the Capacity of an ATS Sector}

For this purpose, the parameters determining the frequency of the work tasks $(k, T, B$ and $Q)$ are selected from geometric characteristics for the ATS sector under consideration and Air Traffic. $G_{b}, \tau_{t}, \tau_{r}, P$ and $\tau_{c}$ have empirical values.

The workload is calculated by the macroscopic model for an ATS sector with a volume $Q=10000 \mathrm{NM}^{3}$, height $H=10000 \mathrm{ft}$ and average aircraft sector flight time $-T=1200 \mathrm{~s}$. An average rate of convergence $380 \mathrm{kt}$ is adopted, with randomly located aircraft, all flying at a speed $V_{m}=460 \mathrm{kt}$.

Model Parameters: $G_{b}=0,1 ; \quad \tau_{c}=50 \mathrm{~s} ; \quad \tau_{r}=2 \mathrm{~s}$; $\tau_{t}=15 \mathrm{~s} ; \quad P=300 \mathrm{~s} ; \quad T=1200 \mathrm{~s}, \quad M_{h}=7 \mathrm{~nm} ;$ $M_{v}=1000 \mathrm{ft} ; E\left[V_{21}\right]=380 \mathrm{kt} ; Q=10000 \mathrm{NM}^{3}$.

The graph in Fig. 1 shows the workload intensity of the four categories of tasks as a function of the number of aircraft. 


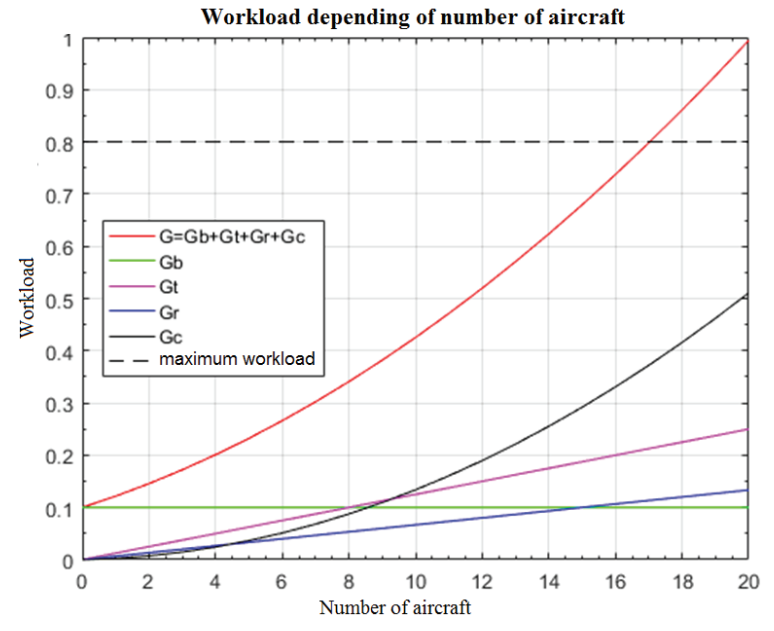

Fig. 1. Workload Intensity depending on the number of aircraft.

For the maximum workload Gm, a value of $80 \%$ of an hour of ATCO operational work is assumed, based on the basic tasks performed and the presence of an automated air traffic management system. From Fig. 1 it follows that the sum total of the work tasks reaches a value of $\mathrm{Gm}$ at $80 \%$ when traffic reaches 17 aircraft (density of 17 aircraft for a volume of $10000 \mathrm{NM}^{3}$ ).

\subsection{Analytical relation between the calculated capacity and the volume of the ATS sector}

The workload from recurring tasks and such related to conflicts, depends directly on the volume of the sector. The boundaries of the sector are extended along the main direction of the air traffic flow, in order to reduce the workload from transition tasks. For this purpose, three sectors are defined with a ratio of 1:1, 1:2 and 1:4. A relation can be found between the volume of the sector and its longest side $a$. For a rectangular sector with a height of $H$, side $a$ and $a$ ratio between the sides 1:u the formula is as follows:

$$
Q=u a^{2} H
$$

If the air traffic flow is along the length of the sector and $\mathrm{Lm}$ is the average distance, which an aircraft flies through the sector, the capacity of the sector can be calculated from its volume and the ratio between the length and width:

$$
\begin{gathered}
L_{m}=u a ; \\
T=\frac{L_{m}}{V_{m}} .
\end{gathered}
$$

The results from equations (9), (10) и (11) are presented in Fig. 2 with the correlation between the capacity and volume of the ATS sector by using the parameters from Fig. 1.

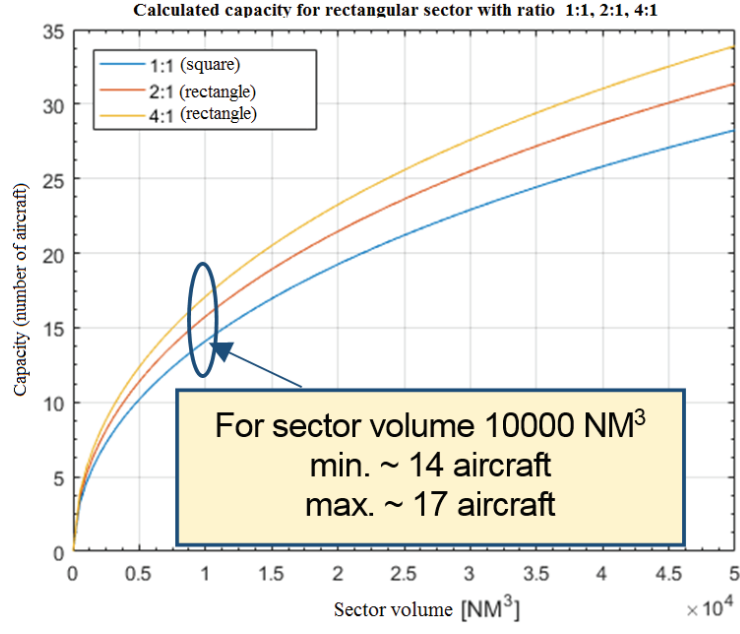

Fig. 2. Calculated capacity of rectangular sectors with a ratio of $1: 1$ (square), 2:1 and 4:1 depending on the volume.

For sectors with a volume of $10000 \mathrm{NM}^{3}$ the graph shows that the calculated capacity increases from 14 aircraft for a square to 17 aircraft for a rectangle with a ratio of $4: 1$. In conclusion, for a sector with a maximum allowed workload, the maximum density of the air traffic decreases with the increase of the volume.

\section{Results from the calculation of the capacity of sector configurations}

When observing configurations of three and four sectors, united into one, the ATC team has to provide services in a relatively big airspace. For that reason configurations $[V A, V C, V D],[V B, V C, V D]$ and $[V A, V B, V C, V D]$ have the following values for parameters $\tau_{r}=3 \mathrm{~s}$ and $P=200 \mathrm{~s}$. In all other configurations: $V A, V B, V D$, $[V A, V C],[V B, V D]$ and $[V C, V D]$, the following values are applicable $\tau_{r}=2 \mathrm{~s}$ and $P=300 \mathrm{~s}$.

Table 1 presents the results from the calculation of the different configurations, on the basis of the defined parameters of every configuration and gives the number of aircraft every ATC has to provide service to

\begin{tabular}{|c|c|c|c|c|c|c|c|}
\hline $\begin{array}{c}\text { Sector } \\
\text { Configuration }\end{array}$ & 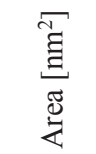 & 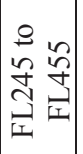 & 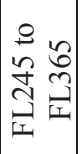 & 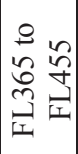 & 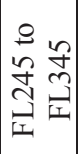 & 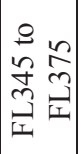 & 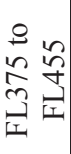 \\
\hline VA & 4648 & 14 & 12 & 13 & 12 & 8 & 11 \\
\hline VB & 5106 & 14 & 12 & 13 & 12 & 8 & 11 \\
\hline VD & 5656 & 17 & 14 & 15 & 14 & 9 & 13 \\
\hline VA\&VC & 7170 & 19 & 15 & 15 & 15 & 10 & 14 \\
\hline VB\&VD & 10762 & 21 & 18 & 19 & 18 & 12 & 17 \\
\hline VC\&VD & 8178 & 20 & 17 & 18 & 17 & 11 & 16 \\
\hline VA\&VC\&VD & 12826 & 20 & 18 & 18 & 18 & 12 & 17 \\
\hline VB\&VC\&VD & 13284 & 17 & 16 & 17 & 16 & 12 & 16 \\
\hline $\begin{array}{c}\text { VA\&VB\&VC } \\
\& V D\end{array}$ & 17932 & 21 & 19 & 20 & 19 & 14 & 18 \\
\hline
\end{tabular}
simultaneously.

Table 1. Calculated capacity for sector configuration. 
In Table 2 the calculated air traffic density is presented for every configuration.

Table 2. Results for air traffic density for an airspace with a volume of $10000 \mathrm{NM}^{3}$.

\begin{tabular}{|c|c|c|c|c|c|c|c|}
\hline $\begin{array}{c}\text { Sector } \\
\text { Configuration }\end{array}$ & 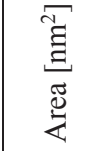 & 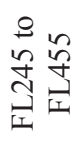 & 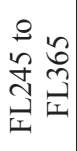 & 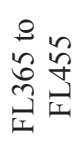 & 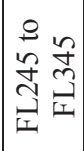 & 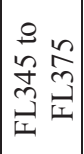 & 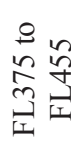 \\
\hline VA & 4648 & 8 & 13 & 18 & 15 & 34 & 17 \\
\hline VB & 5106 & 7 & 11 & 17 & 14 & 31 & 16 \\
\hline VD & 5656 & 8 & 12 & 17 & 15 & 32 & 17 \\
\hline VA\&VC & 7170 & 7 & 10 & 14 & 12 & 28 & 14 \\
\hline VB\&VD & 10762 & 5 & 8 & 11 & 10 & 22 & 11 \\
\hline VC\&VD & 8178 & 7 & 10 & 14 & 12 & 27 & 14 \\
\hline VA\&VC\&VD & 12826 & 4 & 7 & 9 & 8 & 18 & 10 \\
\hline VB\&VC\&VD & 13284 & 3 & 6 & 8 & 7 & 18 & 9 \\
\hline $\begin{array}{c}\mathrm{VA} \& \mathrm{VB} \& \mathrm{VC} \\
\& \mathrm{VD}\end{array}$ & 17932 & 3 & 5 & 7 & 6 & 15 & 7 \\
\hline
\end{tabular}

Fig. 3 shows the results graphically for sector configurations $V A$ and $V C$ and $V D$.

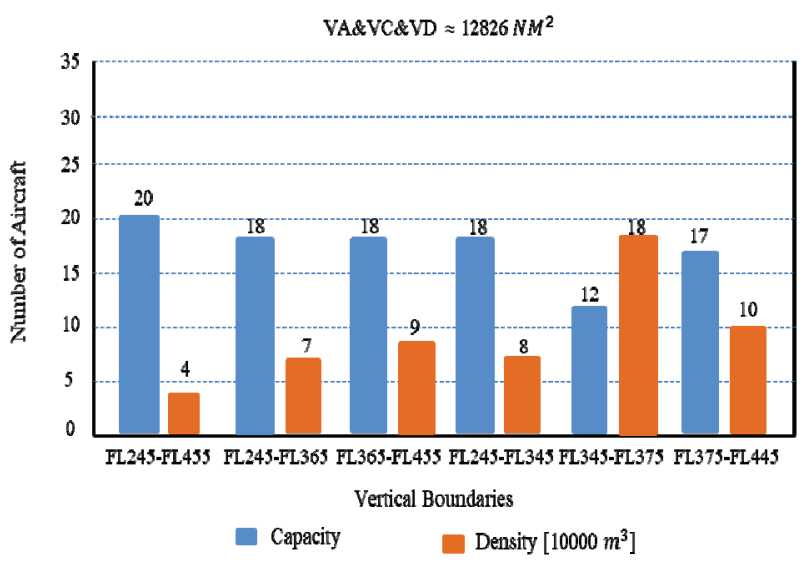

Fig. 3. Results for the capacity and density for $10000 \mathrm{NM}^{3}$.

A conclusion can be made that in order to increase the maximum number of aircraft for a certain sector configuration, the maximum allowed air traffic density has to be increased. This can be achieved when the airspace is divided into smaller sectors, in order to provide service to air traffic with a greater density. As a result, within the whole airspace the maximum number of aircraft increases.

\section{Conclusion}

The present work on capacity determination of sectoral airspace configurations is a study of the workload of the ATC. Determining the capacity of sector configurations is a subtask of the unified task of increasing the efficiency of airspace. The results obtained can be used to estimate the workload of the ATC. The formulas shown may be used for automated implementation of basic ATM features that provide benefits for flight safety and efficiency of air transport. For a maximum workload value, $\mathrm{Gm}$ can be assumed $80 \%$ with an automated air traffic management system. Determining the workload of the ATC has practical significance for Strategic capacity planning of the sectoral configurations.

\section{References}

1. ICAO, doc. 9882, Manual on air traffic management system requirements, (2008)

2. P. Petrov, Automation of management of the air traffic - part one, Publishing House of Technical University - Sofia, (2013)

3. A. Majumdar, W. Ochieng, G. McAuley, J. Lenzi, C. Lepadatu, The factors affecting airspace capacity in Europe: A framework methodology based on cross sectional time-series nalysis using simulated controller workload data, (2004)

4. BULATSA, AIP Republic of Bulgaria - ENR 6.2-3, (2016)

5. R. Jaurena, Guide for the application of a common methodology to estimate airport and ATC sector capacity for the SAM region, Lima, Peru, July (2009)

6. F. Dowling, EEC Note No. 4/98 - Sector capacity assessment for Dublin ACC, Eurocontrol, February (1998) 